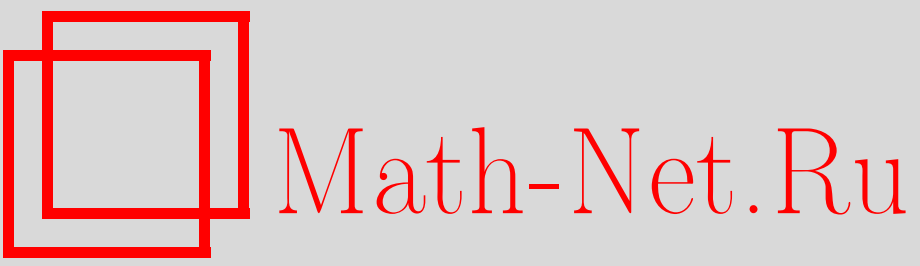

А. А. Арсеньев, Резонансы и туннелирование в квантовой проволоке, ТМФ, 2006, том 147, номер 1, 92-102

DOI: https://doi.org/10.4213/tmf2025

Использование Общероссийского математического портала Math-Net.Ru подразумевает, что вы прочитали и согласны с пользовательским соглашением

http://www.mathnet.ru/rus/agreement

Параметры загрузки:

IP : 54.174 .149 .18

26 апреля 2023 г., 15:53:32

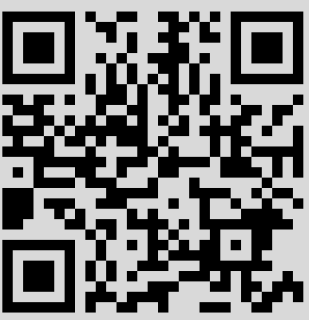


ТЕОРЕТИЧЕСКАЯ

И МАТЕМАТИЧЕСКАЯ

ФИЗИКА

Том 147, № 1

апрель, 2006

2006 г.

\author{
А. А. Арсеньев*
}

\title{
РЕЗОНАНСЫ И ТУННЕЛИРОВАНИЕ В КВАНТОВОЙ ПРОВОЛОКЕ
}

Рассматривается связанная с моделью баллистической проводимости задача математической теории рассеяния. Исследуется модель, описывающая распространение заряда в квантовой проволоке. Предполагается, что носитель заряда имеет спин, и учитывается спин-орбитальное взаимодействие Рашбы. Изучаются резонансы проводимости, порожденные слабым взаимодействием квантовой проволоки с квазистационарным состоянием параллельно присоединенной квантовой точки или с туннелированием через последовательно присоединенную квантовую точку. Такой квантовой точкой обычно является управляющий элемент. Приведены достаточные условия для пространственной симметрии системы, при выполнении которых квазистационарое состояние квантовой точки порождает резонанс проводимости. Предполагается, что проводимость связана с матрицей рассеяния формулой Ландауэра.

Ключевые слова: резонанс, квантовая проволока, баллистическая проводимость.

\section{1. ВВЕДЕНИЕ}

Изложим на неформальном уровне содержание работы. Рассмотрим систему, которая состоит из квантовой проволоки и квантовой точки. Спектр гамильтониана квантовой проволоки непрерывен, спектр гамильтониана квантовой точки дискретен. Если квантовая проволока и квантовая точка не взаимодействуют, то собственные значения гамильтониана квантовой точки являются погруженными собственными значениями гамильтониана системы "квантовая проволока и квантовая точка". При включении слабого взаимодействия между квантовой точкой и квантовой проволокой погруженные собственные значения превращаются в резонансы: полюсы матрицы расеяния с малой мнимой частью. Формула Ландауэра связывает проводимость с модулем коэффициента прохождения в матрице рассеяния. Однако модули элементов матрицы рассеяния в общем случае как функции спектрального параметра могут и не испытывать скачка в окрестности полюса матрицы рассеяния (тривиальный пример - матрица рассеяния для радиального уравнения Шредингера, модуль которой тождественно равен единице). Возникает вопрос: при каких

* Московский государственный университет, Москва, Россия. E-mail: arsenev@afrodita.phys.msu.su, arsenev@math356.phys.msu.su 
условиях порожденный малым возмущением погруженного собственного значения полюс матрицы рассеяния действительно порождает резонанс проводимости? В работе [1] отмечено, что в полупроводниковых гетероструктурах при рассеянии на неоднородностях (в ситуации, которая, по-видимому, хорошо описывается моделью баллистической проводимости) резонанс проводимости наблюдается только в том случае, если система обладает пространственной симметрией. В одной из первых работ по теории туннельного диода [2] наблюдение о связи между пространственной симметрией системы и резонансом проводимости сделано на основе анализа точно решаемой задачи о рассеянии на двойном прямоугольном барьере. В работе [3] предложена математическая модель, которая учитывает роль пространственной симметрии при образовании резонанса проводимости из квазистационарного состояния. На основе предложенной в статье [3] модели в работе [4] в приближении сильной связи, баллистической проводимости и без учета спина носителей заряда получены условия резонанса проводимости в квантовой проволоке, которая взаимодействует с параллельно или последовательно присоединенной квантовой точкой. В данной работе мы рассматриваем ту же физическую задачу, что и в работе [4], но на этот раз мы используем другую математическую модель и учитываем спин носителей заряда.

Основные результаты работы сформулированы в теоремах 1 и 2 . С точки зрения физики эти результаты могут быть интерпретированы так.

1. Если гамильтониан квантовой точки коммутирует с отображением $l_{1}$ (см. формулу (15)), то слабое взаимодействие квантовой проволоки с параллельно присоединенной квантовой точкой приводит к запиранию квантовой проволоки на энергиях, совпадающих с энергиями собственных состояний квантовой точки.

2. Если гамильтониан последовательно присоединенной квантовой точки коммутирует с отображением $l_{2}$ (см. формулу (15)), то слабое взаимодействие квантовой точки с квантовой проволокой приводит к туннелированию через квантовую точку волн, энергия которых совпадает с энергией собственных состояний квантовой точки.

Условия туннелирования в рассматриваемой нами модели не затрагивают спиновых переменных и совпадают с условиями туннелирования в модели сильной связи без учета спина, а условия запирания затрагивают спиновые переменные.

\section{2. ОПИСАНИЕ МОДЕЛИ КВАНТОВОЙ ПРОВОЛОКИ}

В приближении баллистической проводимости свободная динамика носителей заряда в квантовой проволоке с учетом спина и спин-орбитального взаимодействия Рашбы в общепринятых обозначениях [5] описывается гамильтонианом

$$
H_{\text {wire }}=\frac{1}{2 m}\left(p_{x}^{2}+p_{y}^{2}\right)+V(x)+\frac{\alpha}{\hbar}\left(\sigma_{x} p_{y}-\sigma_{y} p_{x}\right),
$$

где $\sigma_{x}, \sigma_{y}$ - матрицы Паули, $V(x)$ - растущий на бесконечности потенциал, который ограничивает в плоскости $x 0 y$ занятую током область, $\alpha$ - константа спинорбитального взаимодействия Рашбы. Предполагается, что ток распространяется вдоль оси $y$ и обуславливающее спин-орбитальное взаимодействие Рашбы электри- 
ческое поле направлено перпендикулярно плоскости $x 0 y$. Мы считаем, что внешнего магнитного поля нет.

Мы будем предполагать, что единицы измерения выбраны так, что гамильтониан (1) имеет вид

$$
H_{\text {wire }}=-\frac{\partial^{2}}{\partial x^{2}}-\frac{\partial^{2}}{\partial y^{2}}+V(x)-i a\left(\sigma_{x} \frac{\partial}{\partial y}-\sigma_{y} \frac{\partial}{\partial x}\right)
$$

где $a$ - вещественная константа. Будем считать потенциал $V(x)$ бесконечно дифференцируемым, неотрицательным, симметричным $(V(x)=V(-x))$ и достаточно быстро растущим на бесконечности.

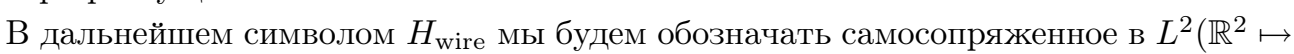
$\left.\mathbb{C}^{2}, d x d y\right)$ расширение оператора, заданного формулой $(2)$ на финитных функциях.

С точки зрения физики гамильтониан (2) описывает динамику носителей заряда только в малой окрестности энергии Ферми, поэтому в нашей задаче имеет смысл ограничиться построением локальной матрицы рассеяния. Это позволит нам не отвлекаться на несущественные в нашей задаче математические детали. Заметим, что локализация гамильтониана является стандартным приемом в квантовой оптике. Мы локализуем гамильтониан (2) на спектральный интервал $\left(\lambda_{0}-\delta, \lambda_{0}+\delta\right)$, который выбирается исходя из двух соображений: интервал $\left(\lambda_{0}-\delta, \lambda_{0}+\delta\right)$ должен содержать только одно простое собственное значение гамильтониана квантовой точки $\lambda_{0}$, и на этом интервале выполнено предположение 1 (см. ниже). Ясно, что наши построения имеют физический мысл только тогда, когда интервал $\left(\lambda_{0}-\delta, \lambda_{0}+\delta\right)$ расплолжен вблизи уровня Ферми.

Приступим к построению спектральной функции $E(\lambda)$ гамильтониана $H_{\text {wire }}$ в окрестности точки $\lambda_{0}$. В дальнейшем мы считаем, что $\left|\lambda-\lambda_{0}\right|<\delta$, где $\delta$ достаточно мало.

Будем искать решение уравнения

$$
H_{\text {wire }} u=\lambda u
$$

в виде

$$
u(x, y)=e^{-i k y} \phi(x, k)
$$

Для функции $\phi$ получим уравнение

$$
L_{k} \phi=\left(\lambda-k^{2}\right) \phi
$$

где

$$
L_{k}=-\frac{\partial^{2}}{\partial x^{2}}+V(x)-a k \sigma_{x}+i a \sigma_{y} \frac{\partial}{\partial x}
$$

Пусть $\phi_{j}(x, k)$ - нормированные в $L^{2}\left(\mathbb{R}^{1}, d x\right)$ собственные функции оператора $L_{k}$ и $\mu_{j}(k)$ - отвечающие им собственные значения оператора $L_{k}$ :

$$
L_{k} \phi_{j}(x, k)=\mu_{j}(k) \phi_{j}(x, k), \quad j=0, \ldots
$$


Мы считаем, что собственные функции действительны, каждому собственному значению соответствует только одна собственная функция, но среди собственных значений могут быть равные. Мы не считаем, что собственные значения упорядочены по возрастанию. В дальнейшем мы будем рассматривать собственные значения как функции параметра $k$, и нумерация собственных значений может зависеть от рассматриваемой окрестности параметра $k$.

Рассмотрим уравнение

$$
k^{2}+\mu_{j}(k)=\lambda, \quad \lambda \in\left(\lambda_{0}-\delta, \lambda_{0}+\delta\right) .
$$

Пусть $k\left(\lambda_{0}, \alpha\right), \quad \mu_{j(\alpha)}\left(k\left(\lambda_{0}, \alpha\right)\right), \quad 1 \leqslant \alpha \leqslant q,-$ решения уравнения (4) на полуоси $k>0$ при $\lambda=\lambda_{0}$. Мы считаем, что $\alpha \neq \beta$, если $k\left(\lambda_{0}, \alpha\right) \neq k\left(\lambda_{0}, \beta\right)$.

Сделаем следующее предположение.

ПрЕДПОЛОЖЕНИЕ 1. При любом $\lambda \in\left(\lambda_{0}-\delta, \lambda_{0}+\delta\right)$ уравнение (4), рассматриваемое как уравнение относительно $k$, на интервале $k>0$ имеет решение только для набора индексов $j \in\{j(\alpha), 1 \leqslant \alpha \leqslant q\}$, и для каждого $j=j(\alpha)$ из этого набора решение $k(\lambda, \alpha)$ уравнения (4) единственно и таково, что

$$
\frac{d k(\lambda, \alpha)}{d \lambda}>0, \quad \lambda \in\left(\lambda_{0}-\delta, \lambda_{0}+\delta\right) .
$$

Мы предположим, что

$$
k 2(\alpha) \stackrel{\text { def }}{=} k\left(\lambda_{0}+\delta, \alpha\right)>k 1(\alpha) \stackrel{\text { def }}{=} k\left(\lambda_{0}-\delta, \alpha\right)>0 .
$$

Далее мы будем считать, что параметр $k$ изменяется в области

$$
\bigcup_{1 \leqslant \alpha \leqslant q}\{k ; k 1(\alpha)<|k|<k 2(\alpha)\} .
$$

ЛЕмма 1. Если число $\mu_{j}(k)$ и фучнкиия $\phi_{j}(x, k)$ удовлетворяют уравнению (3), то число $\mu_{j}(k)$ и функиия $i \sigma_{y} \phi_{j}(x, k)$ удовлетворяют уравнению $(3)$, в которм сделана замена $k \rightarrow-k$.

ДоКАЗАТЕЛЬСТво. Умножим обе части уравнения (3) на $\sigma_{y}$ и используем антикоммутативность матриц $\sigma_{x}$ и $\sigma_{y}$.

В пространстве $L^{2}\left(\mathbb{R}^{2} \mapsto \mathbb{C}^{2}, d x d y\right)$ определим оператор

$$
P_{j}: P_{j} f(x, y ; k)=\left(\int \phi_{j}\left(x^{\prime}, k\right) \cdot f\left(x^{\prime}, y\right) d x^{\prime}\right) \phi_{j}(x, k),
$$

где - - линейное по второму аргументу скалярное произведение в $\mathbb{C}^{2}$.

Определим оператор

$$
\mathcal{P}_{\text {wire }}=E\left(\lambda_{0}+\delta\right)-E\left(\lambda_{0}-\delta\right)
$$


ЛЕмма 2. Если справедливо предположение 1 , то спектралъный проектор $\mathcal{P}_{\text {wire }}$ на интервал $\left(\lambda_{0}-\delta, \lambda_{0}+\delta\right)$ для оператора $H_{\text {wire }}$ дается формулой

$$
\mathcal{P}_{\text {wire }} f(x, y)=\frac{1}{2 \pi} \sum_{\alpha} \int\left(\int_{k 1(\alpha) \leqslant|k| \leqslant k 2(\alpha)} e^{-i k\left(y-y^{\prime}\right)} P_{j(\alpha)} f\left(x, y^{\prime} ; k\right) d k\right) d y^{\prime} .
$$

ДокАЗАТЕльство. Для вычисления спектральной функции оператора $H_{\text {wire }}$ нам нужно знать его резольвенту. В уравнении для неизвестной функции $g$

$$
\left(\lambda-H_{\text {wire }}\right) g=f, \quad|\operatorname{Im} \lambda|>0,
$$

сделаем преобразование Фурье по $y$, используем известную формулу для функции Грина дифференциального оператора с чисто дискретным спектром и предположение 1 , а потом совершим обратное преобразование Фурье и найдем $g$. Так мы получим резольвенту. Затем используем известную формулу, которая связывает спектральную функцию и резольвенту, и получим формулу (8).

Положим по определению

$$
H_{\text {wire }, \text { loc }}=\mathcal{P}_{\text {wire }} H_{\text {wire }}
$$

Из леммы 2 следует

Лемма 3. Справедливо равенство

$$
\begin{aligned}
H_{\text {wire }, \text { loc }} f(x, y)=\sum_{\alpha} & \frac{1}{2 \pi} \int\left(\int_{k 1(\alpha) \leqslant|k| \leqslant k 2(\alpha)} e^{-i k\left(y-y^{\prime}\right)}\left(k^{2}+\mu_{j(\alpha)}(k)\right) \times\right. \\
& \left.\times P_{j(\alpha)} f\left(x, y^{\prime} ; k\right) d k\right) d y^{\prime} .
\end{aligned}
$$

Если предположение 1 выполнено, то формула (10) задает локализацию гамильто-

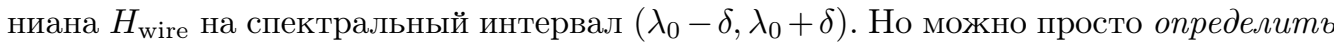
гамильтониан проволоки формулой (10). С точки зрения физики это будет означать, что мы пренебрегаем процессами с энергиями, которые сильно отличаются от энергии Ферми. Такое предположение нам кажется разумным и согласованным с точностью модели баллистической проводимости, поэтому мы в дальнейшем по определению будем считать гамильтонианом проволоки оператор, заданный формулой (10).

Определим пространство

$$
\mathcal{H}_{\text {wire }}=\mathcal{P}_{\text {wire }} L^{2}\left(\mathbb{R}^{2} \mapsto \mathbb{C}^{2}, d x d y\right)
$$

Определенный формулой $(10)$ оператор $H_{\text {wire,loc }}$ самсопряжен в пространстве $\mathcal{H}_{\text {wire }}$, и пространство $\mathcal{H}_{\text {wire }}$ приводит оператор $H_{\text {wire,loc }}$.

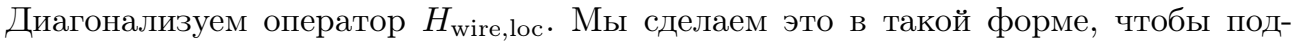
черкнуть аналогию с теорией потенциального рассеяния и дифракции и облегчить применение изложенного в работе [3] формализма. 
Пусть $\Omega=\{ \pm 1, \pm 2, \ldots, \pm q\}$ - набор чисел. Этими числами в дальнейшем мы будем параметризовать распространяющиеся по проволоке моды, причем знак числа $\omega= \pm \alpha$ мы возьмем совпадающим со знаком проекции импульса распространяющейся моды на ось $y$. Заметим, что в силу леммы 1 каждому значению спектрального параметра $\lambda$ отвечают отличающиеся знаком два значения параметра $k$. Превратим множество $\Omega$ в пространство с мерой, приписав каждой точке $\omega \in \Omega$ меру 1 . Пусть

$$
h=L^{2}\left(\left(\left(\lambda_{0}-\delta, \lambda_{0}+\delta\right) \mapsto L^{2}(\Omega)\right), d \lambda\right)
$$

- гильбертово пространство квадратично-интегрируемых функций на интервале $\left(\lambda_{0}-\delta, \lambda_{0}+\delta\right)$ со значениями в $L^{2}(\Omega)$. Скалярное произведение в $h$ задается формулой

$$
\langle f, g\rangle_{h}=\int_{\lambda_{0}-\delta<\lambda<\lambda_{0}+\delta}\left(\sum_{\alpha} \overline{f(\alpha, \lambda)} g(\alpha, \lambda)\right) d \lambda .
$$

Пусть

$$
U: \mathcal{H}_{\text {wire }} \mapsto h
$$

- отображение, которое действует по формуле

$$
\begin{aligned}
(U f)(\lambda, \omega)= & \left(\frac{1}{2 \pi} \frac{d k(\lambda,|\omega|)}{d \lambda}\right)^{1 / 2} \times \\
& \times \int e^{i \operatorname{sign}(\omega) k(\lambda,|\omega|) y} \phi_{j(|\omega|)}(x, k(\lambda,|\omega|)) f(x, y) d x d y
\end{aligned}
$$

Из формулы (8) следует, что отображение $U$ унитарно в $\mathcal{H}_{\text {wire }}$ и обратное отображение $U^{-1}$ есть интегральный оператор в $h$ с ядром

$$
\begin{aligned}
U^{-1}(x, y \mid \lambda, \omega)= & \left(\frac{1}{2 \pi} \frac{d k(\lambda,|\omega|)}{d \lambda}\right)^{1 / 2} \times \\
& \times e^{-i \operatorname{sign}(\omega) k(\lambda,|\omega|) y} \phi_{j(|\omega|)}(x, \operatorname{sign}(\omega) k(\lambda,|\omega|)) .
\end{aligned}
$$

Из формулы (10) и определения $k(\lambda, \alpha)$ как корня уравнения (4) следует, что опеpaтop $U H_{\text {wire,loc }} U^{-1}$ в пространстве $h$ есть оператор умножения на $\lambda$, и поэтому

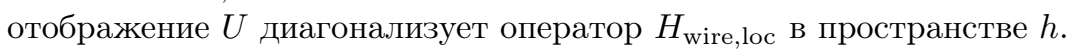

Функции

$$
\begin{aligned}
e(x, y, \lambda, \omega)= & \left(\frac{1}{2 \pi} \frac{d k(\lambda,|\omega|)}{d \lambda}\right)^{1 / 2} \times \\
& \times e^{-i \operatorname{sign}(\omega) k(\lambda,|\omega|) y} \phi_{j(|\omega|)}(x, \operatorname{sign}(\omega) k(\lambda,|\omega|))
\end{aligned}
$$

суть нормированные на $\delta$-функцию обобщенные собственные функции непрерывного спектра для оператора $H_{\text {wire,loc }}$.

ЛЕмма 4. Отображения

$$
l_{1}: \psi(x, y) \mapsto \sigma_{y} \bar{\psi}(x, y), \quad l_{2}: \psi(x, y) \mapsto \bar{\psi}(x,-y)
$$

коммутируют с гамильтонианом $H_{\text {wire }}$.

Это утверждение проверяется вычислением.

4 Теоретическая и математическая физика, т. 147, № 1, 2006 г. 


\section{3. РАССЕЯНИЕ НА КВАНТОВОЙ ТОЧКЕ}

Для описания взаимодействия квантовой проволоки с квантовой точкой мы используем формализм “систем $2 \times 2$ " (см. литературные ссылки в работе [3]).

Сначала мы рассмотрим модель взаимодействия квантовой проволоки с параллельно присоединенной квантовой точкой.

Пусть $\mathcal{H}_{\text {dot }} \subset L^{2}\left(\mathbb{R}^{2} \mapsto \mathbb{C}^{2}, d x d y\right)$ - конечномерное пространство,

$$
\mathcal{H}_{\text {dot }} \perp \mathcal{H}_{\text {wire }}
$$

Пусть $H_{\text {dot }}-$ самосопряженный оператор в $\mathcal{H}_{\text {dot }}$ и $\lambda_{0}-$ простое собственное значение оператора $H_{\mathrm{dot}}$. Мы предположим, что интервал $\left(\lambda_{0}-\delta, \lambda_{0}+\delta\right)$ не содержит других собственных чисел оператора $H_{\mathrm{dot}}$.

Пусть $V_{\text {int }}$ - самосопряженный ядерный оператор в пространстве

$$
\mathcal{H}=\mathcal{H}_{\text {dot }} \oplus \mathcal{H}_{\text {wire }}
$$

Мы предположим, что оператор $V_{\text {int }}$ мал. Понятие малости мы формализуем так: мы будем рассматривать последовательность операторов $\left(V_{\mathrm{int}}\right)_{n}$, которая удовлетворяет условиям

$$
\left(V_{\text {int }}\right)_{0}=0, \quad\left\|\left(V_{\text {int }}\right)_{n}\right\| \rightarrow 0, \quad n \rightarrow \infty .
$$

Норма в формуле $(16)$ - ядерная норма в $L^{2}\left(\mathbb{R}^{2} \mapsto \mathbb{C}^{2}, d x d y\right)$. Доопределим нулем операторы $H_{\text {wire,loc }}, H_{\text {dot }}$ на все пространство $\mathcal{H}$ и положим

$$
H_{n}=H_{\text {wire, loc }}+H_{\text {dot }}+\left(V_{\text {int }}\right)_{n}
$$

Оператор $H_{n}$ мы будем интерпретировать как гамильтониан квантовой проволоки, которая взаимодействует с квантовой точкой.

Пусть $S\left(H_{n}, H_{0}\right)$ - оператор рассеяния для пары $H_{n}, H_{0}$. Упорядочим базис $(14)$ : сначала поставим функции $e(x, y, \lambda, \omega)$ с $\omega=1,2, \ldots$, а потом с $\omega=-1,-2, \ldots$. В пространстве $h$ в упорядоченном таким образом базисе (14) оператор $S\left(H_{n}, H_{0}\right)$ задается $(2 q \times 2 q)$-матричной функцией

$$
\lambda \rightarrow S\left(H_{n}, H_{0}\right)(\lambda)=\left(\begin{array}{ll}
A_{n}(\lambda) & B_{n}(\lambda) \\
C_{n}(\lambda) & D_{n}(\lambda)
\end{array}\right)
$$

Элементы $(q \times q)$-миноров $A_{n}(\lambda), D_{n}(\lambda)$ в формуле $(17)$ отождествляются с коэффициентами прохождения, элементы миноров $B_{n}(\lambda), C_{n}(\lambda)-$ с коэффициентами отражения. Элементы миноров $A_{n}(\lambda), D_{n}(\lambda)$ мы будем обозначать символами $t_{i, j ; n}$, $1 \leqslant i, j \leqslant 2 q$, а элементы миноров $B_{n}(\lambda), C_{n}(\lambda)$ - символами $r_{i, j ; n}, \quad 1 \leqslant i, j \leqslant 2 q$. Заметим, что в работе [3] символом $t_{n}$ обозначены другие величины: матричные элементы $T$-матрицы. Обозначения фиксированы традицией, и мы надеемся, что это не приведет к недоразумениям.

Наша задача свелась к изучению поведения функции (17) в окрестности точки $\lambda_{0}$ при $n \rightarrow \infty$. Для этого мы воспользуемся предложенной в работе [3] схемой. 
Положим

$$
\begin{aligned}
\mathcal{H}_{1} & =\mathcal{H}_{\text {dot }}, \\
\mathcal{H}_{2}^{-} & =P_{\text {wire }} L^{2}\left(\mathbb{R}^{2} \mapsto \mathbb{C}^{2}, e^{|y|} d x d y\right), \\
\mathcal{H}_{2}^{+} & =\text {пополнение в } L^{2}\left(\mathbb{R}^{2} \mapsto \mathbb{C}^{2}, e^{-|y|} d x d y\right) \text { пространства } \mathcal{H}_{\text {wire }}
\end{aligned}
$$

Пространства $\mathcal{H}_{1}, \mathcal{H}_{2}^{-}, \mathcal{H}_{2}$ и $\mathcal{H}_{2}^{+}$удовлетворяют условиям $1-3$ работы [3].

Аналитичность отображения $U^{-1}$ и резольвенты $R\left(\lambda H_{\text {wire,loc }}\right)$ следует из условия (5) настоящей работы. Условия 4-7 работы [3] в рассматриваемой нами ситуации мы заменим на

ПрЕДПОЛОЖЕНИЕ 2. Операторъ $H_{n}$ при $0<n<\infty$ не имеют собственнъх значений на интервале $\left(\lambda_{0}-\delta, \lambda_{0}+\delta\right)$.

Это предположение означает, что у взаимодействующей системы "квантовая проволока и квантовая точка" нет связанных состояний с энергиями в интервале $\left(\lambda_{0}-\right.$ $\left.\delta, \lambda_{0}+\delta\right)$. Заметим, что условия 4-7 в работе [3] нужны только для доказательства этого предположения.

Условие 8 работы [3] будет следовать из следующего предположения.

ПРЕДПОЛОЖЕНИЕ 3. Операторъ $H_{\mathrm{dot}} u\left(V_{\mathrm{int}}\right)_{n}$ коммутируют с отображениeм $l_{1}$.

Условие 9 работы [3] будет выполнено, если будет выполнено предположение 3 и отображение $p$ будет задано формулой

$$
p=p_{1}: p_{1}(\omega)=-\omega .
$$

Теперь заметим, что в рассматриваемой нами модели при $n=\infty$ квантовая точка и квантовая проволока не взаимодействуют, матрица рассеяния есть единичная матрица, и поэтому при $n=\infty$ миноры $A_{n}(\lambda), D_{n}(\lambda)$ в формуле $(17)$ суть единичные матрицы, а миноры $B_{n}(\lambda), C_{n}(\lambda)$ равны нулю, и поэтому

$$
r_{2 q+1-j, j ; \infty}(\lambda) \equiv 0, \quad \lambda \in\left(\lambda_{0}-\delta, \lambda_{0}+\delta\right), \quad 1 \leqslant j \leqslant 2 q .
$$

Лемма 6 работы [3] и равенство (26) из той же работы с учетом равенства (19) могут теперь быть сформулированы в виде следующей теоремы.

ТЕОРема 1. 1. При достаточно большом $n$ в окрестности точки $\lambda_{0}$ существует полюс $\lambda_{n}$ матрицы рассеяния (17), причем

$$
\operatorname{Im} \lambda_{n}<0, \quad n<\infty, \quad \lambda_{n} \rightarrow \lambda_{0}, \quad n \rightarrow \infty .
$$

2. Если последовательность действительных чисел $\nu_{n}$ удовлетворяет условию

$$
\left|\nu_{n}-\operatorname{Re} \lambda_{n}\right|=O\left(\left|\operatorname{Im} \lambda_{n}\right|^{1+\epsilon}\right), \quad \epsilon>0, \quad n \rightarrow \infty,
$$

то для коэффициентов отражения матрицы рассеяния (17) будет выполнено соотношение

$$
\sum_{1 \leqslant j \leqslant 2 q}\left|r_{2 q+1-j, j ; n}\left(\nu_{n}\right)\right| \rightarrow 2, \quad n \rightarrow \infty .
$$


Введем функцию

$$
\sigma_{n}(\lambda)=\sum_{1 \leqslant i, j \leqslant 2 q}\left|t_{i, j ; n}(\lambda)\right|^{2} .
$$

Если есть только один канал рассеяния $(q=1)$, то величина $\sigma_{n}(\lambda)$ с точностью до размерного множителя совпадает с проводимостью; в общем случае проводимость зависит от плотности состояний в источнике и стоке, но все же величина $\sigma_{n}(\lambda)$ может дать некоторое представление о величине проводимости. Из соотношения (20) следует, что

$$
\liminf _{n \rightarrow \infty}\left(\sum_{1 \leqslant j \leqslant 2 q}\left|r_{2 q+1-j, j ; n}\left(\nu_{n}\right)\right|^{2}\right) \geqslant \frac{2}{q} .
$$

Из унитарности матрицы рассеяния и формулы (22) следует, что

$$
\limsup _{n \rightarrow \infty} \sigma_{n}\left(\nu_{n}\right) \leqslant 2\left(q-\frac{1}{q}\right) .
$$

Соотношение (23) и является математической формулировкой явления "запирания" квантовой проволоки на резонансных энергиях $\nu_{n}$, так как для всех $\lambda \neq \lambda_{0}$

$$
\lim _{n \rightarrow \infty} \sigma_{n}(\lambda)=2 q
$$

Теперь рассмотрим взаимодействие квантовой проволоки с последовательно присоединенной квантовой точкой. Мы имеем следующую конструкцию. Квантовая проволока идет из $-\infty$ в точку $L$, между точками $L$ и $R$ расположена квантовая точка, а далее квантовая проволока идет из точки $R$ в $+\infty$. С математической точки зрения нам будет удобно считать, что квантовая проволока идет из $-\infty$ в $+\infty$, а соответствующую разность гамильтонианов добавить в гамильтониан квантовой точки. Мы будем считать, что фазовое пространство системы "квантовая проволока и квантовая точка" есть прямая сумма конечномерного пространства $\mathcal{H}_{\text {out }} \subset L^{2}\left(\mathbb{R}^{2} \mapsto \mathbb{C}^{2}, d x d y\right)$ и пространства $\mathcal{H}_{\text {wire }}$, а гамильтониан системы "квантовая проволока и квантовая точка" есть оператор

$$
H_{n}=\left(\begin{array}{cc}
\left(H_{\text {dot }, \text { out }}\right)_{n} & \left(V_{\text {int }}\right)_{n} \\
\left(V_{\text {int }}\right)_{n}^{\star} & H_{\text {wire,loc }}+\left(H_{\text {dot, int }}\right)_{n}
\end{array}\right) .
$$

Символом $H_{0}$ мы обозначим оператор

$$
H_{0}=\left(\begin{array}{cc}
0 & 0 \\
0 & H_{\text {wire,loc }}
\end{array}\right) \text {. }
$$

Мы будем считать, что выполнены следующие предположения.

ПрЕДПОЛОЖЕнИЕ 4. Операторъ $\left(H_{\mathrm{dot}, \mathrm{out}}\right)_{n},\left(H_{\mathrm{dot}, \mathrm{int}}\right)_{n}$ в бормуле $(24)-$ самосопряженные ядерные операторы и при $n \rightarrow \infty$ в ядерной норме сходятся $\kappa$ операторам $\left(H_{\mathrm{dot}, \mathrm{out}}\right)_{\infty},\left(H_{\mathrm{dot}, \mathrm{int}}\right)_{\infty}$. Oператоры $\left(V_{\mathrm{int}}\right)_{n}-$ ядерные и в ядерной норме сходятся при $n \rightarrow \infty$ к оператору $\left(V_{\mathrm{int}}\right)_{\infty}$. 
ПреДПОЛОЖЕНИЕ 5. Оператор $H_{n}$ в формуле (24) удовлетворяет сбормулированному выше предположению 2.

ПРЕДПОЛОЖЕНИЕ 6. Гамилътониан $H_{\infty}$, который описывает систему из последовательно присоединенной квантовой точки и не взаимодействующими с ней участками $(-\infty, L),(R,+\infty)$ квантовой проволоки, имеет погруженное в непрерывный спектр простое собственное значение $\lambda_{0}$

ПРЕДПОЛОЖЕНИЕ 7. При $n=\infty$ (когда нет взаимодействия) матрица рассеяния для операторов $H_{\infty}, H_{0}$ имеет вид

$$
S\left(H_{\infty}, H_{0}\right)(\lambda)=\left(\begin{array}{cc}
0 & B_{\infty}(\lambda) \\
C_{\infty}(\lambda) & 0
\end{array}\right) .
$$

ПРЕДПОЛОЖЕНИЕ 8. Операторъ $H_{n}$ в бормуле (24) коммутируют с отображением $l_{2}$ из формуль (15).

В качестве отображения $p$ из работы [3] для гамильтониана системы из квантовой проволоки и последовательно присоединенной квантовой точки нужно взять отображение

$$
p=p_{2}: p_{2}(\omega)=\omega .
$$

Дальнейшие рассуждения аналогичны приведенным выше для случая параллельно присоединенной квантовой точки и почти дословно повторяют соответствующие рассуждения для случая модели сильной связи [4]. Окончательный результат мы сформулируем в виде теоремы.

ТЕОРема 2. 1. При достаточно большом $n$ в окрестности точки $\lambda_{0}$ существует полюс $\lambda_{n}$ матрицы рассеяния для операторов $H_{n}, H_{0}$, причем

$$
\operatorname{Im} \lambda_{n}<0, \quad n<\infty, \quad \lambda_{n} \rightarrow \lambda_{0}, \quad n \rightarrow \infty .
$$

2. Если последовательность действительных чисел $\nu_{n}$ удовлетворяет условию

$$
\left|\nu_{n}-\operatorname{Re} \lambda_{n}\right|=O\left(\left|\operatorname{Im} \lambda_{n}\right|^{1+\epsilon}\right), \quad \epsilon>0, \quad n \rightarrow \infty,
$$

то для коэффициентов прохождения матрицы рассеяния операторов $H_{n}, H_{0}$ будет выполнено соотношение

$$
\lim _{n \rightarrow \infty}\left(\sum_{1 \leqslant j \leqslant 2 q}\left|t_{j, j ; n}\left(\nu_{n}\right)\right|\right)=2 .
$$

Из соотношения (25) следует, что

$$
\liminf _{n \rightarrow \infty} \sigma_{n}\left(\nu_{n}\right) \geqslant \frac{2}{q}
$$

Соотношение (26) означает, что на энергиях $\nu_{n}$ частицы резонансно туннелируют через последовательно присоединенную квантовую точку, так как для всех $\lambda \neq \lambda_{0}$

$$
\lim _{n \rightarrow \infty} \sigma_{n}(\lambda)=0 \text {. }
$$


В заключение мы заметим, что локализация гамильтониана нам была нужна только для упрощения доказательств утверждений общего характера об аналитичности резольвенты, матрицы рассеяния, существования полюсов матрицы рассеяния и т.д. Содержательная часть проведенного анализа состоит в выяснении связи между резонансами амплитуды рассеяния, симметрией гамильтониана (которая выражается в том, что гамильтониан коммутирует со специального вида сопряжениями эти сопряжения описывались отображением $l$ ) и симметрией амплитуды рассеяния, которая выражается в симметрии матрицы рассеяния в базисе из собственных функций непрерывного спектра свободного гамильтониана. В нашем случае эта симметрия описывалась отображением $p$. С методической точки зрения основным результатом данной работы является пример анализа связи между симметрией задачи и резонансом амплитуды рассеяния для задачи со спином.

Благодарности. Автор глубоко благодарен рецензенту за ценные библиографические ссылки и внимательное рецензирование работы.

\section{Список литературы}

[1] C. W. J. Beenaker, H. von Houten, Solid State Phys., 44 (1991), 1; cond-mat/0412664.

[2] B. Ricco, M. Ya. Azbel, Phys. Rev. B, 29 (1984), 1970.

[3] А. А. Арсеньев, TMФ, 136 (2003), 507.

[4] А. А. Арсеньев, ТМФ, 141 (2004), 100.

[5] Ю. А. Бычков, Э.И. Рашба, Писъма в ЖЭТФ, 39 (1984), 66; Ү.А. Bychkov, E. I. Rashba, J. Phys. C, 17 (1984), 6039; M. Governale, U. Zülicke, Rashba spin splitting in quantum wires, cond-mat/0407036; T. Koga, J. Nitta, H. Takayanagi, S. Datta, Phys. Rev. Lett., 88 (2002), 126601.

Поступила в редакцию 19.IX.2005 г., после доработки 31.Х.2005 г. 